\title{
Laparoscopic Hemihysterectomy for Uterus Didelphys Following Laparotomy
}

\author{
Areiyu Zhang, MD, Faten F. Abdelhafez, MD, James Liu, MD, Mohamed A. Bedaiwy, MD, PhD \\ Division of Reproductive Endocrinology and Infertility, University of British Columbia, \\ Vancouver, Canada (Drs. Zang, Bedaiwy). \\ Department of Obstetrics and Gynecology, University Hospitals Case Medical Center, Case Western Reserve University, \\ Cleveland, Ohio, USA (Drs. Abdelhafez, Bedaiwy, Liu).
}

\begin{abstract}
Introduction: Müllerian anomalies can be challenging to diagnose. We describe a patient with uterus didelphys and cervical atresia who presented with a symptomatic pelvic mass that was incompletely diagnosed as an endometrioma. Consequently, it was inadequately treated at the time of laparotomy, necessitating a laparoscopic hemihysterectomy for definitive management.

Case Description: A 17-year-old nulligravida presented with abdominal pain and pelvic pressure. Computed tomographic imaging showed an $18-\mathrm{cm}$ pelvic mass and left renal agenesis. The patient underwent an exploratory laparotomy with resection of a large endometrioma and left salpingo-oophorectomy. During the operation, an enlarged left uterine horn was observed and was kept intact. She made an uneventful recovery, but returned 6 months later with pelvic pain and pressure. Ultrasonography and hysterosalpingogram diagnosed uterus didelphys with left cervical atresia causing an obstructed hemiuterus. Laparoscopic hemihysterectomy with extensive adhesiolysis was performed, and the patient's symptoms resolved.

Discussion: Endometrioma is rare in adolescents, and its presence should raise suspicion of a müllerian anomaly. Laparoscopic management, while safe and feasible, is more challenging in the setting of dense adhesions from a prior laparotomy. Preoperative magnetic resonance imaging is recommended in adolescents who present with chronic pelvic pain and a symptomatic pelvic mass, to rule out a congenital anomaly and avoid a needless second surgery.
\end{abstract}

Key Words: Cervical atresia, Endometrioma, Hemihysterectomy, Müllerian anomaly, Uterus didelphys.

Citation Zhang A, Abdelhafez FF, Liu J, Bedaiwy MA. Laparoscopic hemihysterectomy for uterus didelphys following laparotomy. CRSLS e2014.00250. DOI: 10.4293/JSLS.2014.00250.

Copyright (C) 2015 by SLS, Society of Laparoendoscopic Surgeons. This is an open-access article distributed under the terms of the Creative Commons Attribution-Noncommercial-ShareAlike 3.0 Unported license, which permits unrestricted noncommercial use, distribution, and reproduction in any medium, provided the original author and source are credited.

Conflicts of interest: no disclosures.

Address correspondence to: Mohamed Bedaiwy MD, PhD, Reproductive Endocrinology and Infertility, Department of Obstetrics and Gynaecology, Faculty of Medicine, The University of British Columbia, D415A-4500 Oak Street, Vancouver, BC V6H 3V4 Canada. Telephone: 604-97-2588, Fax: 604-875-2987, E-mail: bedaiwymmm@yahoo.com

\section{INTRODUCTION}

Müllerian tract anomalies are found in up to $10 \%$ of the general population. ${ }^{1-6}$ Uterus didelphys arises from a fusion defect of the müllerian tract. Each hemiuterus usually has its own patent cervix draining into the vagina. If either of these cervices is atretic, the respective hemiuterus will not drain appropriately, and hematometra will develop. There are only a few reports of this unusual variant in the literature. ${ }^{7,8}$ Because of their common embryologic ori- gins, associated urologic abnormalities on clinical presentation of müllerian anomalies are high. We present a case involving a 17-year-old who had a missed diagnosis of uterus didelphys with left cervical atresia at the time of laparotomy for excision of an endometrioma, necessitating a laparoscopic hemihysterectomy 6 months later.

The first laparoscopic surgical excision of a rudimentary horn of a unicornuate uterus was reported by Canis and colleagues $^{9}$ in 1990. Since then, 28 cases have been reported, yet none of these patients had a history of major 
surgical abdominal exploration. ${ }^{10-18}$ We show that the laparoscopic approach for an obstructed uterine horn is safe and feasible after a midline laparotomy. This case emphasizes the importance of preoperative imaging in making an accurate diagnosis in an adolescent presenting with a symptomatic pelvic mass. Coexisting endometriosis is often seen with obstructing müllerian anomalies. An endometrioma in an adolescent should raise suspicion of an associated congenital reproductive tract anomaly.

\section{CASE REPORT}

A 17-year-old nulligravida with a body mass index of 32 was referred to our tertiary university hospital with abdominal pain and pelvic pressure. Computed tomographic (CT) imaging showed an 18-cm complex adnexal mass and a congenitally absent left kidney. The patient had regular menses and reported occasional dysmenorrhea. On speculum examination, one vagina and one cervix were seen. Cystoscopy revealed a single ureteral orifice on the right side. An exploratory laparotomy was performed through a midline incision. Removal of the large mass required extensive lysis of adhesions and dissection from the sigmoid colon. A left salpingo-oophorectomy was also performed. During the surgical exploration, the left kidney and ureter were confirmed to be absent. Incidentally, an enlarged left hemiuterus was discovered that was left intact. Frozen pathology of the mass revealed an endometrioma, confirmed on final pathology. The patient's course in the hospital was uneventful, and she was discharged home on postoperative day 3 .

Six months after the operation, the patient again presented with chronic pelvic pain and pressure. Pelvic ultrasonography revealed hematometra in the obstructed left hemiuterus. A hysterosalpingogram demonstrated a normal right hemiuterus with no filling defects and a patent right fallopian tube. The patient consented to a laparoscopic excision of the left hemiuterus with possible conversion to laparotomy.

The left upper quadrant was chosen for the site of primary trocar insertion. Peritoneal entry was achieved without complication, and a $10-\mathrm{mm}$ camera was inserted. Pneumoperitoneum was obtained. Accessory ports were placed on the right side of the abdominal wall. Thick bands of dense, fatty adhesions were seen along the left side of the abdominal cavity, extending to the pelvic sidewall to an enlarged left hemiuterus. The right hemiuterus was normal in size and free of adhesions (Figure 1A). The rectosigmoid colon was densely adherent to the superior and posterior aspect of the left hemiuterus. Careful adhe- siolysis with blunt and sharp dissection and bipolar electrosurgery with PKS Cutting Forceps (Gyrus Medical, Maple Grove, Minnesota) was necessary to isolate the hemiuterus for removal. Next, the left round ligament was ligated to gain access to the retroperitoneal space. Consistent with the findings of the previous laparotomy, the left ureter was congenitally absent. The left uterine artery was identified at its origin through dissection of the anterior division of the internal iliac artery. The artery was skeletonized and ligated at its point of entry into the hemiuterus (Figure 1B), followed by ligation of the left uterosacral ligament. The bladder flap was developed, thereby completing the isolation of the hemiuterus for removal (Figure 1C). Scissors and electrosurgery were used to develop a plane between the thick myometrial connection between the hemiuteri. During the dissection, the cavity of the left horn was entered and the hematometra was evacuated. Next, the hemiuterus was dissected to the level of the vaginal vault and completely amputated. The small partial-thickness myometrial defect on the right hemiuterus was repaired in a single layer with a running 2-0 V-Loc suture (Covidien, Mansfield, Massachusetts). The uterine specimen was removed through morcellation. The pelvis was then irrigated and excellent hemostasis was achieved (Figure 1D). The patient was discharged home on the same day and made an uneventful postoperative recovery. Final pathology confirmed the presence of myometrium and active endometrium. The patient had complete resolution of her pelvic pain.

\section{DISCUSSION}

This is the first reported case of laparoscopic hemihysterectomy for obstructed uterus didelphys after a major abdominal exploration. Only one case of laparoscopic excision of a uterine horn following laparotomy has been reported in the literature thus far. In that case, a small (35- $\mathrm{mm})$ atrophic rudimentary horn was removed via a 2-cm McBurney incision through a previous appendectomy incision. ${ }^{16}$ There is no question that the laparoscopic approach is more technically challenging after a midline laparotomy. In addition, the patient had a history of endometriosis and a large hemiuterus with high vascularity. ${ }^{13}$ Although GnRH agonists have been recommended to reduce the size of rudimentary horns with hematometra and to decrease vascularity, ${ }^{10,11}$ they were not used in our patient. Instead, the surgery was scheduled at the end of her menses to facilitate better definition of the planes of dissection. Left upper quadrant access through Palmer's point was used as a primary peritoneal entry site to avoid injury to bowel or other intraperitoneal structures that may 


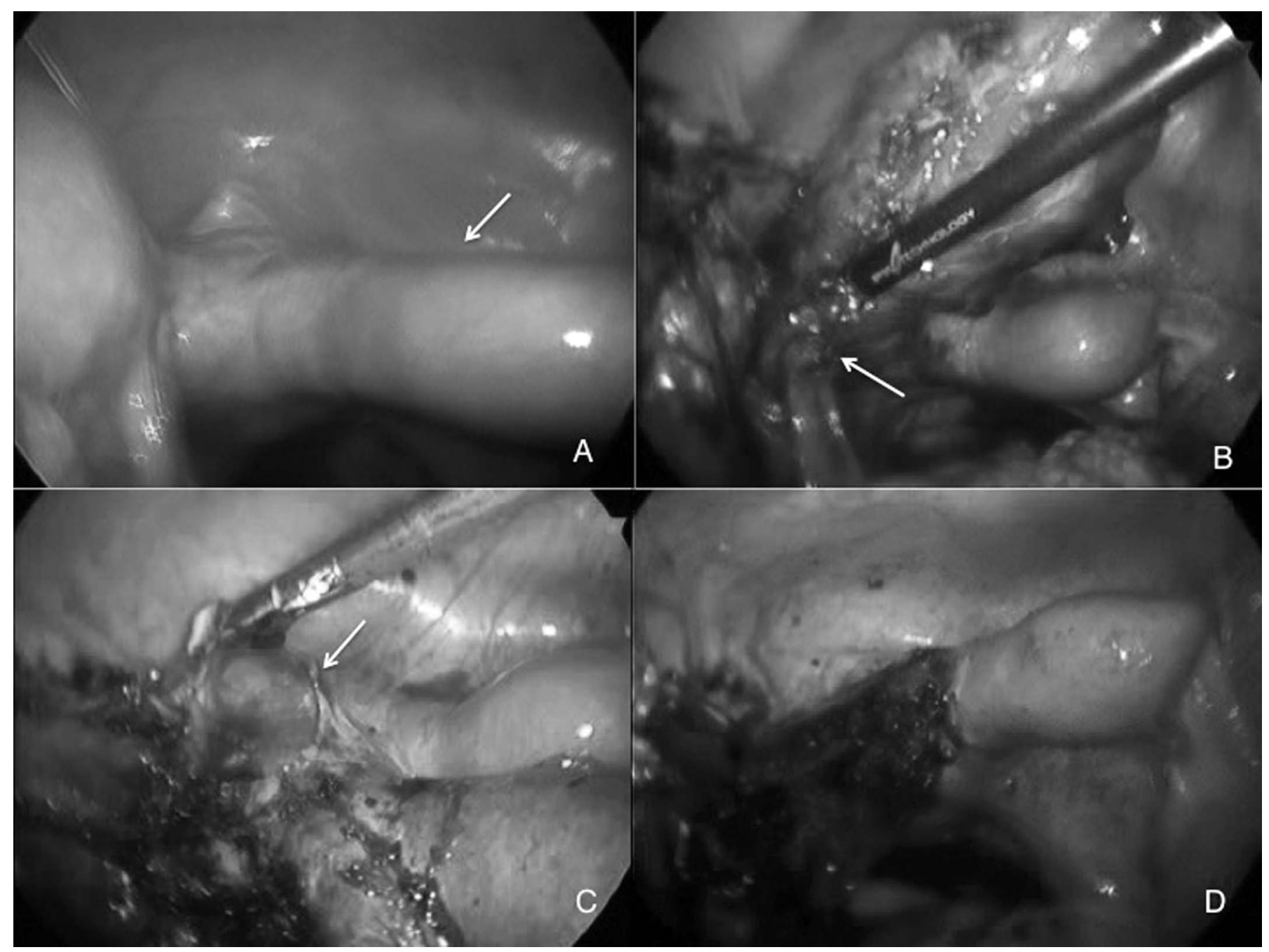

Figure 1. A, Visualization of uterus didelphys with an enlarged left hemiuterus adherent to the rectosigmoid colon. B, Ligation of the left uterine artery after the left hemiuterus has been extensively dissected. C, Creation of the bladder flap. The left hemiuterus has been devascularized, as evidenced by its duskiness. D, Visualization of the pelvis after removal of the left hemiuterus.

have been adherent to the midline scar. This primary access has been recommended for both conventional and robotic laparoscopic procedures in patients with a history of prior abdominal surgeries. ${ }^{19-22}$

Regrettably for the patient, a second laparoscopy was necessary that could have been avoided altogether had the müllerian anomaly been correctly diagnosed at the time of initial presentation. Endometrioma is rare in adolescents. Young patients with endometriosis diagnosed on laparoscopy often have ASRM (American Society of Reproductive Medicine) stage I or II disease and atypical clear and red lesions. ${ }^{23}$ The presence of endometrioma in an adolescent should raise suspicion of an obstructing müllerian anomaly. The incidence of coexisting endome- triosis with congenital anomalies of the reproductive tract varies in the literature but can be as high as $77 \% .{ }^{24}$ In a series of 15 patients younger than 20 years with endometriosis, $40 \%$ also had a müllerian anomaly. ${ }^{25}$ In contrast, Goldstein and colleagues $^{26}$ found that, of 74 cases of endometriosis diagnosed at laparoscopy in adolescents, only $11 \%$ were associated with a müllerian anomaly. Once the outflow tract obstruction has been corrected, the endometriosis also resolves without further treatment. ${ }^{27}$

Our case highlights the importance of ruling out an obstructive congenital anomaly in an adolescent presenting with chronic pelvic pain and suspected endometrioma, as this population would benefit from early surgical intervention. There should be a low threshold for obtaining 
additional preoperative imaging, such as magnetic resonance imaging (MRI) or 3-dimensional ultrasonography, as these modalities are highly sensitive and specific for the diagnosis of müllerian anomaly. ${ }^{28}$ MRI offers information on tissue composition, as it can differentiate between endometrium and myometrium by signal intensity on T2weighted images. ${ }^{29}$ Three-dimensional ultrasonography is a less expensive alternative to an MRI and accurately visualizes both uterine contour and intracavitary anomalies. ${ }^{30}$ In this case, the only imaging the patient had before the first laparotomy was a CT scan. Use of multimodal imaging would have better characterized the patient's pelvic mass in relation to the complex müllerian anomaly. Definitive management of the endometrioma and the left uterine horn containing hematometra could then have been achieved during the same procedure.

In conclusion, endometrioma is rare in adolescents, and its presence should raise suspicion of a coexisting müllerian anomaly. Preoperative investigations with MRI or 3-dimensional ultrasonography are recommended, to ensure the correct diagnosis and avoid an unnecessary second operation.

\section{References:}

1. Byrne J, Nussbaum-Blask A, Taylor WS, et al. Prevalence of Müllerian duct anomalies detected at ultrasound. Am J Med Genet. 2000;94:9-12.

2. Jurkovic D, Gruboeck K, Tailor A, Nicolaides KH. Ultrasound screening for congenital uterine anomalies. Br J Obstet Gynaecol. 1997;104:1320-1321.

3. Maneschi F, Zupi E, Marconi D, Valli E, Romanini C, Mancuso S. Hysteroscopically detected asymptomatic müllerian anomalies: prevalence and reproductive implications. $J$ Reprod Med. 1995;40:684-688.

4. Nahum GG. Uterine anomalies: how common are they, and what is their distribution among subtypes? J Reprod Med. 1998;43:877-887.

5. Chan YY, Jayaprakasan K, Zamora J, Thornton JG, RaineFenning N, Coomarasamy A. The prevalence of congenital uterine anomalies in unselected and high-risk populations: a systematic review. Hum Reprod Update. 2011;17:761-771.

6. Dreisler E, Sorensen SS. Müllerian duct anomalies diagnosed by saline contrast sonohysterography: prevalence in a general population. Fertil Steril. 2014;102:525-529.

7. Saleh M, Badawy SZ. Unilateral non-communicating cervical atresia in a patient with uterus didelphys and unilateral renal agenesis. J Pediatr Adolesc Gynecol. 2010;23:e137-e140.

8. Lee CL, Wang CJ, Swei LD, Yen CF, Soong YK. Laparoscopic hemi-hysterectomy in treatment of a didelphic uterus with a hypoplastic cervix and obstructed hemivagina. Hum Reprod. 1999;14:1741-1743.

9. Canis M, Wattiez A, Pouly JL, Mage G, Manhes H, Bruhat MA. Laparoscopic management of unicornuate uterus with rudimentary horn and unilateral extensive endometriosis: case report. Hum Reprod. 1990;5:819-820.

10. Mais V, Guerriero S, Ajossa S, Piras B, Melis GB. Endosonographic diagnosis, pre-operative treatment and laparoscopic removal with endoscopic stapler of a rudimentary horn in a woman with unicornuate uterus. Hum Reprod. 1994;9:12971299.

11. Fedele L, Bianchi S, Zanconate G, Bergamini V. Laparoscopic removal of the cavitated noncommunicating rudimentary uterine horn: surgical aspects in 10 cases. Fertil Steril. 2005;83: 432-436.

12. Nezhat F, Nezhat C, Bess O, Nezhat CH. Laparoscopic amputation of a noncommunicating rudimentary horn after a hysteroscopic diagnosis: a case study. Surg Laparosc Endosc. 1994; 4:155-156.

13. Falcone T, Gidwani G, Paraiso M, Beverly C, Goldberg J. Anatomical variation in the rudimentary horns of a unicornuate uterus: implications for laparoscopic surgery. Hum Reprod. 1997;12:263-265.

14. Schattman GL, Grifo JA, Birnbaum S. Laparoscopic resection of a noncommunicating rudimentary uterine horn: a case report. J Reprod Med. 1995;40:219-220.

15. Giatras K, Licciardi FL, Grifo JA. Laparoscopic resection of a noncommunicating rudimentary uterine horn. J Am Assoc Gynecol Laparosc. 1997;4:491-493.

16. Perrotin F, Bertrand J, Body G. Laparoscopic surgery of unicornuate uterus with rudimentary uterine horn. Hum Reprod. 1999;14:931-933.

17. Dulemba JF, Midgett WA, Freeman MVR. Laparoscopic management of a rudimentary horn pregnancy. J Am Assoc Gynecol Laparosc. 1996;3:S10-S11.

18. Zapardiel I, Alvarez P, Perez-Medina T, Bajo-Arenas JM. Laparoscopic management of a cavitated noncommunicating rudimentary uterine horn of a unicornuate uterus: a case report. J Med Case Rep. 2010;4:215.

19. Tufek I, Akpinar H, Sevinc C, Kural AR. Primary left upper quadrant (Palmer's point) access for laparoscopic radical prostatectomy. Urol J. 2010;7:152-156.

20. Granata M, Tsimpanakos I, Moeity F, Magos A. Are we underutilizing Palmer's point entry in gynecologic laparoscopy? Fertil Steril. 2010;94:2716-2719.

21. Howard FM, El-Minawi AM, DeLoach VE. Direct laparoscopic cannula insertion at the left upper quadrant. J Am Assoc Gynecol Laparosc. 1997;4:595-600. 
22. Ahmad G, Duffy JM, Philips K, Watson A. Laparoscopic entry techniques. Cochrane Database Syst Rev. 2008; CD006583.

23. Laufer MR, Sanfilippo J, Rose G. Adolescent endometriosis: diagnosis and treatment approaches. J Pediatr Adolesc Gynecol. 2003;16:S3-S11.

24. Olive DL, Henderson DY. Endometriosis and müllerian anomalies. Obstet Gynecol. 1987;69:412-415.

25. Schifrin BS, Erez S, Moore JG. Teenage endometriosis. Am J Obstet Gynecol. 1973;116:973-980.

26. Goldstein DP, deCholnoky C, Leventhal M, Emans SJ. New insights into the old problem of chronic pelvic pain. $J$ Pediatr Surg. 1979;14:675-680.
27. Sanfilippo JS, Wakim NG, Schikler KN, Yussman MA. Endometriosis in association with uterine anomaly. Am J Obstet Gynecol. 1986;154:39-43.

28. Deutch TD, Abuhamad AZ. The role of 3-dimensional ultrasonography and magnetic resonance imaging in the diagnosis of müllerian duct anomalies: a review of the literature. J Ultrasound Med. 2008;27:413-423.

29. Marten K, Vosshenrich R, Funke M, Obenauer S, Baum F, Grabbe E. MRI in the evaluation of müllerian duct anomalies. Clin Imaging. 2003;27:346-350.

30. Wu MH, Hsu CC, Huang KE. Detection of congenital müllerian duct anomalies using three-dimensional ultrasound. J Clin Ultrasound. 1997;25:487-492. 